CERN-TH/98-67

DESY-98-034

DTP-98-02

hep-ph/9803497

\title{
An Analysis of Diffraction in Deep-Inelastic Scattering
}

\author{
J. Bartels ${ }^{a}$, J. Ellis ${ }^{b}$, H. Kowalski ${ }^{c}$ and M. Wüsthoff ${ }^{d}$ \\ ${ }^{a}$ II. Institut für Theoretische Physik, Universität Hamburg, Germany. \\ ${ }^{b}$ Theory Division, CERN, Switzerland \\ ${ }^{c}$ Deutsches Elektronen Synchrotron DESY, Germany \\ ${ }^{d}$ University of Durham, Department of Physics, Durham DH1 3LE, UK
}

\begin{abstract}
We propose a simple parametrization for the deep-inelastic diffractive cross section. It contains the contribution of $q \bar{q}$ production to both the longitudinal and the transverse diffractive structure functions, and of the production of $q \bar{q} g$ final states from transverse photons. We start from the hard region and perform a suitable extrapolation into the soft region. We test our model on the 1994 ZEUS and H1 data, and confront it with the H1 conjecture of a singular gluon distribution.
\end{abstract}

\section{Introduction}

Diffractive events are characterized, in general, by the presence of large rapidity gaps in the hadronic final state that are not exponentially suppressed. These are conventionally ascribed to Pomeron exchange. Diffractive processes in deep-inelastic scattering (DIS) are of particular interest, because the hard photon in the initial state gives rise to the hope that, at least in part, the scattering amplitude can be calculated in perturbative QCD (pQCD). With the increasing amount of data on diffractive DIS [1, 2], we have reached a level of accuracy that provides deeper insight into the nature of the Pomeron and its coupling to partons. Rapidity-gap events make up a sizable fraction of all DIS events, and can only be due to the exchange of some colourless object in the cross channel. The simplest realization of the Pomeron in pQCD is provided by two gluons of opposite color [3]. More detailed models based upon the two-gluon picture have been formulated both for perturbative gluons [4] and for nonperturbative (massive) gluons [5, 6, 7, 8]: for an alternative approach, see [9]. In this paper we take the point of view that perturbative QCD provides a reasonable starting point, as in the more detailed model described in $\llbracket$, 10]. In the present paper, we develop a simple parametrization based on this model, which can easily be compared with experimental data.

The simplest description of diffractive DIS starts from the process of $q \bar{q}$ production. This process is conveniently described in terms of light-cone wave functions [6, 11, 12] in the proton rest frame. The light-cone wave function of the photon contains the information about the dissociation of the fast-traveling photon into partons, long before the interaction with the proton occurs. At the beginning of the scattering process, the photon splits into a quark-antiquark pair. At sufficiently 
large photon virtuality $Q^{2}$, the quark-antiquark pair radiates additional gluons before it reaches the proton at rest. At the time of the interaction, the partonic system is spread over a transverse area which is comparable with the size of the hadron. One expects therefore that the exchanged Pomeron should be close to the usual soft hadronic Pomeron. However, inside the final state of the partonic system, we expect that there are also "hard" configurations, for which the exchanged Pomeron should behave quite differently. These are final states for which the partonic system is confined to small transverse distances. Examples are longitudinally-polarized vector particles [12] and high- $p_{T}$ jets [13, 14. In the inclusive measurement of diffractive final states, one sums over both these small-distance and large-distance configurations. So far there is no theoretical framework which allows one to predict the relative magnitudes of the "soft" and the "hard" components of the diffractive cross section, which must be determined by experiment . Since the cross section for the "soft" component is expected to rise weakly with energy for any fixed mass of the diffractive system, whereas the "hard" part should rise faster, the energy dependence of the diffractive cross section may help to determine the relative sizes of the two components.

In attempting to formulate a model that interpolates between these two components, one finds that perturbative models based upon two-gluon exchange, which are valid a priori only for smallsize final states, allow a smooth extrapolation into the soft region. Thanks to gauge invariance and colour cancellation, one does not encounter infrared singularities in models for $q \bar{q}$ production or $q \bar{q} g$ production, i.e., there is no need for an artificial cutoff. Moreover, the wave-function formalism can be extended to include multi-gluon exchange, which is useful for going beyond the lowest-order two-gluon exchange.

Since the first observation of diffractive DIS at HERA, several attempts have been made to compare the data with QCD-based models. In particular, the concept of the Pomeron structure function and its DGLAP $Q^{2}$ evolution has been applied [16, 17, 18, 2]. In these analyses, the diffractive cross section has been assumed to consist only of leading twist, and the contribution of the longitudinal photon has been disregarded. On the other hand, there is strong evidence that, for small masses of the diffractive system, the longitudinal cross section for $q \bar{q}$ production - although formally of higher twist - is not small compared to the transverse cross section. We therefore feel that a more complete analysis of the HERA data should include the longitudinal cross section, and particular emphasis should be given to the region of small diffractive masses. As a minimal model, one might consider just the production of quark-antiquark pairs, which should dominate the small-mass region. For somewhat larger masses, the production of an extra gluon has to be taken into account. In a future step, one would also have to address the $Q^{2}$ evolution of both the transverse and the longitudinal cross sections.

In this paper we propose and test a simple parametrization of the diffractive cross section that is motivated by the above considerations. Stimulated by perturbative QCD, we make an ansatz that consists of four terms, which model both the transverse and the longitudinal cross sections for $q \bar{q}$ and $q \bar{q} g$ production. By treating the overall strength of these terms and the exponent of the energy dependence as a free parameter, we let the data decide which fractions of the diffractive cross section belong to the "soft" and "hard" parts. After a brief description of the model, we compare this Ansatz with the ZEUS and H1 1994 data, and finally draw a few conclusions.

\footnotetext{
${ }^{1}$ It was pointed out in [15] that the pseudo-rapidity cuts imposed in certain early analyses selected a "hard" component in diffractive DIS, which must also be present, at some level, even in analyses without this a priori selection.
} 


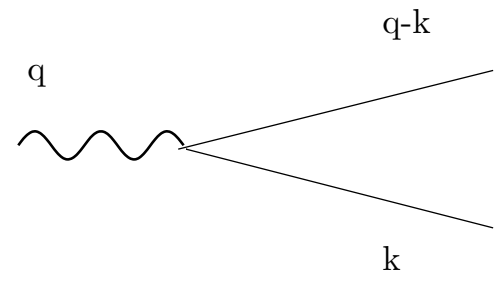

a

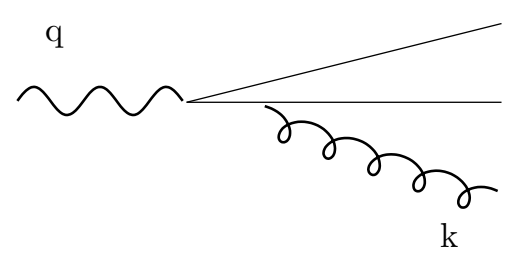

b

Figure 1: Wave function of the photon, including (a) a $q \bar{q}$ component, and (b) a $q \bar{q} g$ component.

\section{A Parametrization for Diffractive DIS}

\subsection{Theoretical Motivation}

The main variables used for the description of diffractive DIS are the total hadronic energy $W$ of the $\gamma^{*}$-proton system and the diffractively-produced mass $M$. In the analysis of the diffractive structure function, it is convenient to use also the variables $\beta$ and $x_{\mathbb{P}}$. In terms of $W$ and $M$, one has $\beta=Q^{2} /\left(M^{2}+Q^{2}\right)$ and $x_{\mathbb{P}}=\left(M^{2}+Q^{2}\right) /\left(W^{2}+Q^{2}\right)$, where we have neglected the proton mass and the momentum transfer $t$. To connect these variables with the Bjorken scaling variable $x_{B}$, we recall that $x_{B}=Q^{2} /\left(W^{2}+Q^{2}\right)$, which immediately leads to $x_{B}=\beta x_{\mathbb{P}}$.

Before describing our model in somewhat more detail, we first make a few general remarks. First, we expect the cross section to be dominated by very small $t$ values. After integration over final-state kinematic variables, the $t$ dependence and the strength of the coupling of the Pomeron to the proton will be combined in the overall normalization. Next, the $\beta$ spectrum and the $Q^{2}$-scaling behavior follow from evolution of the final-state partons, and can most easily be derived from the light-cone wave functions of the incoming photon. This part of our model therefore decouples from the dynamics inside the Pomeron. On the other hand, the energy dependence of the Pomeron,

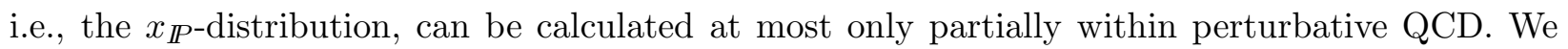
therefore leave it as a free parameter.

We have already indicated that, in the proton rest frame, the light-cone wave function formalism provides a nice intuitive description of diffractive DIS. At leading order, when the photon dissociates into a quark-antiquark pair, we have a single color dipole with a certain momentum distribution given by the corresponding wave function. At higher order, more partons are generated and the initial state can be rather complex. At leading-twist level, however, the basic structure is again a single color dipole: all partons (gluons or quarks) but one are located within a small area in impact-parameter space, i.e., at short relative distances, whereas the remaining single parton is well separated. The localized parton subsystem carries color conjugate to that of the single parton, so that one has again a color-dipole configuration. The short-distance evolution within the parton subsystem factorizes, so one only needs to introduce a wave function for the momentum distribution of the single parton. With this simplification, we end up with two basic structures: a quarkantiquark dipole and a gluon-gluon dipole, the latter appearing only at higher order.

In the case of the elementary quark-antiquark final state, the wave function depends on the helicities of the photon and of the (anti)quark. We define left- and right-handed transverse photons by projecting on the polarization vectors $(1, \mathrm{i})$ and $(1,-\mathrm{i})(\gamma= \pm 1)$, respectively, and the longitudinal 
polarization vector is proportional to the proton momentum $p(\gamma=0)$. For massless quarks, the spin is orientated along the direction of motion or opposite to it $(h= \pm 1)$. As variables for the wave function, we use the Sudakov parameters $k=\alpha q^{\prime}+\beta_{k} p+k_{t}$ with $q^{\prime}=q+x_{B} p$. In the proton rest frame with a fast-moving photon, the parameter $\alpha$ is of the order unity and denotes the momentum fraction of the photon momentum carried by the quark (Figs. 1a, 2), whereas $\beta_{k}$ is small and may be neglected. Using a complex notation for $k_{t}: k=k_{x}+i k_{y}, k^{*}=k_{x}-i k_{y}$, one finds for the transverse photon (see also [11):

$$
\Psi_{h}^{\gamma}\left(\alpha, k_{t}\right)= \begin{cases}-\frac{\sqrt{2}(1-\alpha) k_{t}}{\left|k_{t}\right|^{2}+\alpha(1-\alpha) Q^{2}} & \text { for } \gamma=+1 \text { and } h=+1 \\ \frac{\sqrt{2} \alpha k_{t}}{\left|k_{t}\right|^{2}+\alpha(1-\alpha) Q^{2}} & \text { for } \gamma=+1 \text { and } h=-1 \\ \frac{\sqrt{2} \alpha k_{t}^{*}}{\left|k_{t}\right|^{2}+\alpha(1-\alpha) Q^{2}} & \text { for } \gamma=-1 \text { and } h=+1 \\ -\frac{\sqrt{2}(1-\alpha) k_{t}^{*}}{\left|k_{t}\right|^{2}+\alpha(1-\alpha) Q^{2}} & \text { for } \gamma=-1 \text { and } h=-1\end{cases}
$$

Similarly, for the longitudinal photon one finds:

$$
\Psi_{h}^{\gamma}\left(\alpha, k_{t}\right)=\quad 2 \frac{\alpha(1-\alpha) Q}{\left|k_{t}\right|^{2}+\alpha(1-\alpha) Q^{2}} \quad \text { for } \gamma=0 \text { and } h= \pm 1
$$

Here $Q^{2}, \gamma$ and $h$ denote the virtuality of the photon and the helicities of the photon and the quarks, respectively, and the wave function includes the propagator of the off-shell quark carrying the momentum $k$. In the following, we shall make use of the small- $k_{t}$ behavior of (1) and (2).

To produce a gluon dipole (Fig.1b), we have again to start from the approximation described previously, because a direct coupling of photons to gluons is lacking. In the leading-log $\left(Q^{2}\right)$ approximation, transverse momenta are strongly ordered, which translates into the inverse ordering of distances in impact-parameter space. The quark-antiquark pair with a large transverse momentum is localized in impact-parameter space, and forms an effective "gluon" state conjugate in color to the emitted gluon, which has a smaller transverse momentum and is separated by a large distance from the quark-antiquark pair. In this approach only the transverse photon polarization is of importance, and it determines the hard part of the process, i.e., the effective gluon dipole is independent of whether the photon is right- or left-handed. The wave function of the gluon dipole has the following tensor structure (the indices $\mu, \nu=1,2$, since only the transverse components are involved):

$$
\Psi^{\mu \nu}\left(\alpha, k_{t}\right)=\frac{1}{\sqrt{\alpha(1-\alpha) Q^{2}}} \frac{k_{t}^{2} g_{t}^{\mu \nu}-2 k_{t}^{\mu} k_{t}^{\nu}}{k_{t}^{2}+\alpha(1-\alpha) Q^{2}} .
$$

where $\alpha$ and $k_{t}$ refer to the diffractively-produced gluon. We have written explicitly the $(1-\alpha)$ term, even though the approximation applied here requires $\alpha$ to be much less than 1 , so that $(1-\alpha) \sim 1$. We have introduced this term in order to make manifest the analogy to the previous expression for the quark dipole. Our main interest is, again, the behavior of (3) near $k_{t}=0$.

So far we have discussed the photon wave functions which describe the $q \bar{q}$ or $q \bar{q} g$ state after the splitting of the photon into the quark-antiquark pair. In order to obtain the diffractive scattering amplitude, we have to include the interaction with the proton target. Beginning with the $q \bar{q}$ final 


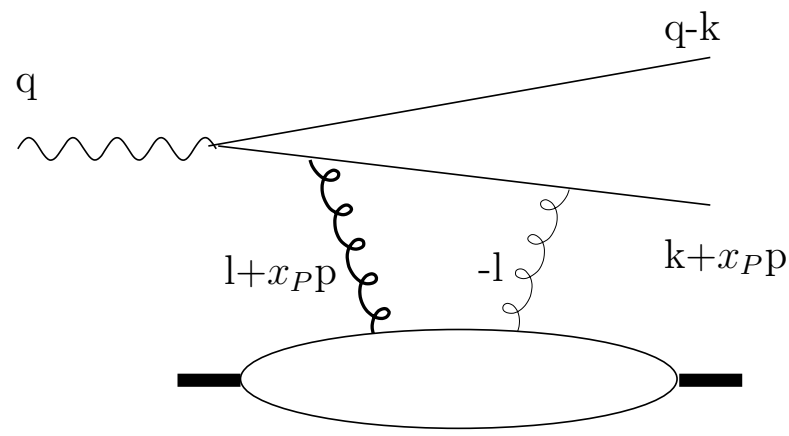

a

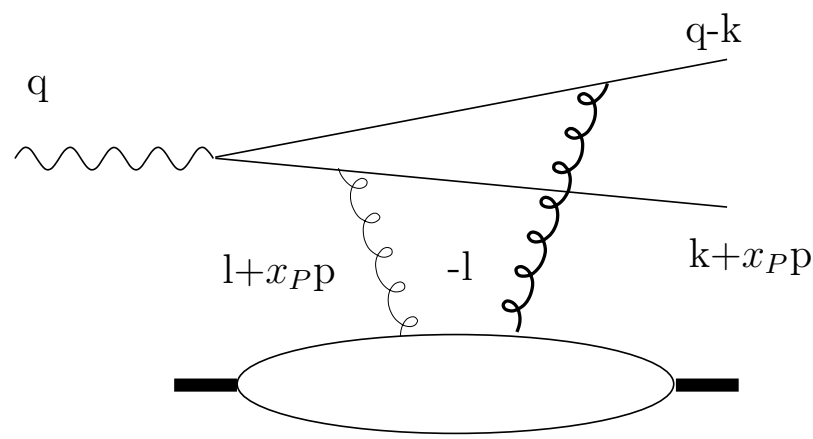

$\mathrm{b}$

Figure 2: Interaction with the proton, modelled in the two-gluon exchange approximation.

state, this interaction is represented in Fig. 2. It is essential that the two gluons couple in all possible ways to the two quarks. In the proton rest frame, the lower quark with momentum $k$ emits a gluon which, after the interaction with the proton, is reabsorbed by one of the two quarks. A more detailed discussion of this two-gluon-exchange model has been given elsewhere [10]. Here we only briefly describe a few main features that we need in order to motivate our parametrization. The coupling of two $t$-channel gluons with zero net color and transverse momenta $l_{t}$ and $-l_{t}$ to the color dipole, i.e., to any wave function of type (1), (2), or (3), can be obtained simply by taking differences of the wave function:

$$
D \Psi:=2 \Psi\left(\alpha, k_{t}\right)-\Psi\left(\alpha, k_{t}+l_{t}\right)-\Psi\left(\alpha, k_{t}-l_{t}\right) \simeq \begin{cases}-l_{t}^{i} l_{t}^{j} \frac{\partial^{2} \Psi\left(\alpha, k_{t}\right)}{\partial k_{t}^{i} \partial k_{t}^{j}} & \text { for } l_{t} \rightarrow 0 \\ 2 \Psi\left(\alpha, k_{t}\right) & \text { for } l_{t} \rightarrow \infty\end{cases}
$$

and then convoluting with a suitable ansatz for the $l_{t}^{2}$ dependence of the Pomeron form factor of the proton.

It is an important feature of our two-gluon model that our simple wave functions (1), (2), (4), together with a suitable ansatz for the Pomeron amplitude, provides an interpolation between the hard and the soft region. In particular, one finds that the transverse polarization belongs to leading twist and is dominated by the aligned-jet configuration, whereas the longitudinal polarization of the photon leads to a higher-twist contribution. In order to see this behavior in the wave-function formalism, we note the relation between the diffractive structure functions $F^{D}$ and our wave functions

$$
F^{D}\left(x_{\mathbb{P}}, \beta, Q^{2}\right) \sim \beta \int d t \int \frac{k_{t}^{2} d^{2} k_{t}}{(1-\beta)^{2}}\left|\int \frac{d^{2} l_{t}}{l_{t}^{2}} D \Psi\left(\alpha, k_{t}\right) \phi\left(l_{t}^{2}, k_{0}^{2} ; x_{\mathbb{P}}\right)\right|^{2}
$$

where $D \Psi$ is taken from $(4)$, and $\phi\left(l_{t}^{2}, k_{0}^{2} ; x_{\mathbb{P}}\right)$ stands for the Pomeron amplitude. Here $k_{0}^{2}$ denotes some hadronic scale which separates the regions of soft and hard QCD: it should not be confused with the QCD factorization scale. The variables $\alpha, \beta, k_{t}^{2}$, and $M^{2}$ are related through

$$
\alpha(1-\alpha) M^{2}=k_{t}^{2},
$$


and $\beta=Q^{2} /\left(Q^{2}+M^{2}\right)$. A simple choice for the $l_{t}$ dependence is [13]

$$
\phi \sim \frac{1}{k_{0}^{2}}\left(\frac{k_{0}^{2}}{l_{t}^{2}}\right)^{\nu\left(l_{t}^{2} / k_{0}^{2}\right)}
$$

where $\nu\left(l_{t}^{2} / k_{0}^{2}\right) \approx 1$ as $l_{t}^{2} \gg k_{0}^{2}$, and $\nu\left(l_{t}^{2} / k_{0}^{2}\right) \rightarrow 0$ as $l_{t}^{2} \rightarrow 0$.

We start in that part of the kinematic region where the parton model of Fig. 2 is most reliable, i.e., the region where the virtuality of the quark with momentum $k$ is large: $k_{t}^{2}+\alpha(1-\alpha) Q^{2}=$ $k_{t}^{2} /(1-\beta)>k_{0}^{2}$ [13]. In this final-state configuration, the $q \bar{q}$ pair has a small transverse size, and the two-gluon Pomeron interacts with the whole system. Consequently, both contributions of Fig. 2 are important, and the simple picture of a "Pomeron structure function", which would be suggested if only Fig. 2a were taken into account, does not apply In this region, the Pomeron amplitude $\phi\left(l_{t}^{2}, k_{0}^{2} ; x_{\mathbb{P}}\right)$ coincides, to a good approximation, with the unintegrated gluon structure function of the proton:

$$
\int^{k_{t}^{2} /(1-\beta)} d l_{t}^{2} \phi\left(l_{t}^{2}, k_{0}^{2} ; x_{\mathbb{P}}\right)=x_{\mathbb{P}} g\left(x_{\mathbb{P}}, k_{t}^{2} /(1-\beta)\right)
$$

(for the extrapolation into the region of smaller $k_{t}$ and for a discussion of the dependence on $x_{\mathbb{P}}$, see below). Going into more detail, let us look into the dependence upon $k_{t}^{2}$ and $\alpha$ at fixed $Q^{2}$. In the $l_{t}$ integral, it suffices to note that the dominant region is $k_{0}^{2}<l_{t}^{2}<k_{t}^{2}+\alpha(1-\alpha) Q^{2}$ : in our example (7), $\phi \sim 1 / l_{t}^{2}$, and the dominant contribution, in fact comes from this kinematic domain. In this region, we approximate $D \Psi$ in (4) by the limit $l_{t} \rightarrow 0$, and obtain:

$$
\begin{aligned}
\int_{k_{0}^{2}}^{k_{t}^{2}+\alpha(1-\alpha) Q^{2}} \frac{d^{2} l_{t}}{l_{t}^{2}} \phi\left(l_{t}^{2}, k_{0}^{2} ; x_{\mathbb{P}}\right) D \Psi & \sim \frac{\alpha(1-\alpha)\left|k_{t}\right| Q^{2}}{\left(k_{t}^{2}+\alpha(1-\alpha) Q^{2}\right)^{3}} x_{\mathbb{P}} g\left(x_{\mathbb{P}}, k_{t}^{2}+\alpha(1-\alpha) Q^{2}\right) \\
& =\frac{\beta(1-\beta)^{2}}{k_{t}^{2}\left|k_{t}\right|} x_{\mathbb{P}} g\left(x_{\mathbb{P}}, k_{t}^{2} /(1-\beta)\right)
\end{aligned}
$$

Inserting this into (5) and making use of relation (6), we find that the integral over $k_{t}^{2}$ (at fixed $\beta$ ) is dominated by the lower limit $k_{0}^{2}$. In terms of the variable $\alpha$, this lower limit corresponds to $\alpha \sim k_{0}^{2} / Q^{2}$ or $1-\alpha \sim k_{0}^{2} / Q^{2}$. The final result for (5) is constant in $Q^{2}$, i.e., it is of leading twist. The end points of the $\alpha$ integral correspond to the aligned configuration: in the center-ofmass system of the quark-antiquark pair (Fig.3), $\alpha$ is related to the scattering angle $\theta$ through $2 \alpha=1-\cos \theta$, and the dominant regions are $\theta=0, \pi$. In other words, starting in the hard region of large transverse momenta, we find that the main contribution comes from the lower end of the $k_{t}$ integral, i.e. we find ourselves pushed into the soft region where our perturbative ansatz for the Pomeron becomes invalid.

A similar argument applied to the longitudinal case shows that the dominance of small $k_{t}^{2}$ (or values of $\alpha$ close to zero or one) is less pronounced: instead of (9), we now have

$$
\begin{aligned}
\int_{k_{0}^{2}}^{k_{t}^{2}+\alpha(1-\alpha) Q^{2}} \frac{d^{2} l_{t}}{l_{t}^{2}} \phi\left(l_{t}^{2}, k_{0}^{2} ; x_{\mathbb{P}}\right) D \Psi & \sim \frac{\alpha(1-\alpha) \sqrt{Q^{2}}\left(\alpha(1-\alpha) Q^{2}-k_{t}^{2}\right)}{\left(k_{t}^{2}+\alpha(1-\alpha) Q^{2}\right)^{3}} x_{\mathbb{P}} g\left(x_{\mathbb{P}}, k_{t}^{2}+\alpha(1-\alpha) Q^{2}\right) \\
& =\frac{\beta(1-2 \beta)(1-\beta)}{k_{t}^{2} Q} x_{\mathbb{P}} g\left(x_{\mathbb{P}}, k_{t}^{2} /(1-\beta)\right)
\end{aligned}
$$

Inserting this into (5), we see that for small $k_{t}^{2}$ the integral diverges only logarithmically. Also, in contrast to the transverse case, the result is of order $1 / Q^{2}$ and hence belongs to nonleading twist.

\footnotetext{
${ }^{2}$ This point has previously been emphasized in 115 .
} 


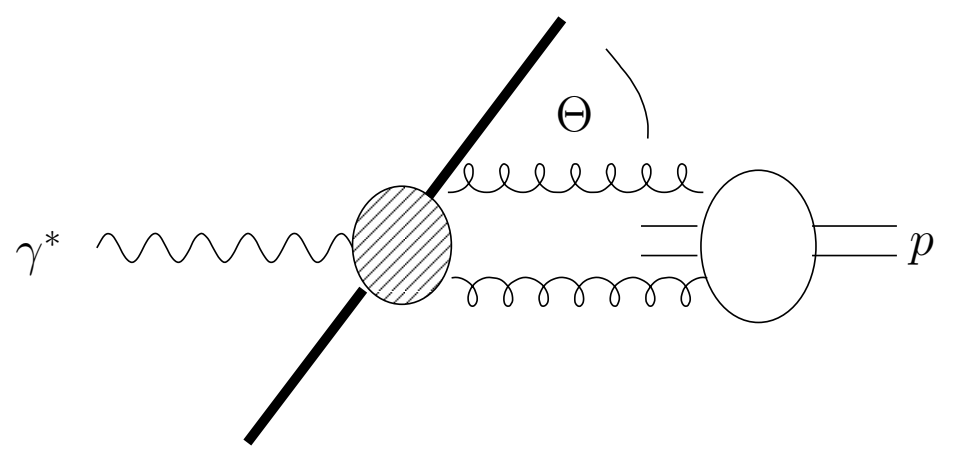

Figure 3: Two-jet production in the $\gamma^{*}-\mathbb{P}$ center-of-mass system

On the other hand, the integration over $k_{t}^{2}$ now yields an additional logarithm in $Q^{2} /\left(4 \beta k_{0}^{2}\right)$, which is absent in the transverse leading-twist case, and slightly compensates for the $Q^{2}$ suppression.

Next we return to the transverse case and take a closer look at the soft region where $k_{t}^{2}+\alpha(1-$ $\alpha) Q^{2}<k_{0}^{2}$, i.e., $k_{t}^{2}<(1-\beta) k_{0}^{2}$ and $\alpha<\beta k_{0}^{2} / Q^{2}$ or $1-\alpha<\beta k_{0}^{2} / Q^{2}$. Now the quark with momentum $k$ in Fig. 2a, before it interacts with the two-gluon Pomeron, is nearly on shell, and one expects the picture of the Pomeron structure function to become valid, i.e., the lower parton in Fig. 2a can be considered more as a "valence" constituent of the Pomeron, and the contribution of Fig. 2b should be less important. This transition can be made explicit by changing the $l_{t}^{2}$ dependence of the Pomeron amplitude, which now can no longer be identified with the unintegrated gluon structure function, in such a way that is gives more weight to the region $l_{t}^{2}<k_{0}^{2}$ : the simple example in eq. (6) leads to $\phi \sim 1 / k_{0}^{2}=$ constant. The correct behavior is obtained simply by taking in (4) $l_{t}^{2}$ much larger than $k_{t}^{2}$, i.e., $l_{t} \rightarrow \infty$ : in this region the first term $2 \Psi\left(\alpha, k_{t}\right)$ dominates, and we end up with

$$
\begin{aligned}
\int \frac{d^{2} l_{t}}{l_{t}^{2}} \phi\left(l_{t}^{2}\right) D \Psi & \sim \frac{\left|k_{t}\right|}{k_{0}^{2}\left(k_{t}^{2}+\alpha(1-\alpha) Q^{2}\right)} \cdot \ln \left(\frac{k_{0}^{2}}{k_{t}^{2}+\alpha(1-\alpha) Q^{2}}\right) \\
& =\frac{1-\beta}{k_{0}^{2}\left|k_{t}\right|} \ln \left(\frac{(1-\beta) k_{0}^{2}}{k_{t}^{2}}\right)
\end{aligned}
$$

which corresponds to the planar diagram Fig. 2a (on the rhs of (11), we have disregarded the dependence upon $x_{\mathbb{P}}$ ). Returning to (5), the integral in $k_{t}^{2}$ can easily be performed and leads to a finite leading-twist result. By similar arguments the longitudinal case is found to be of the order $1 / Q^{2}$.

In summary, with a suitable Ansatz for the $l_{t}$ dependence of the two-gluon Pomeron or, even more simply, with a simple prescription for the $l_{t}$ integral, it is possible to interpolate between the hard region where the parton model applies and the soft region where the aligned jet configuration dominates. For the latter case we seem to have arrived at the same conclusions as Bjorken [21], although with a somewhat different line of arguments. But, as we shall argue further below, there is a new element that we have to take into account, namely the observation of the strong rise of the gluon structure function at small $\mathrm{x}$, which gives a weight to the region of large transverse momenta which is larger than was anticipated before the advent of the HERA data in, for example, [21].

Before we turn to the $x_{\mathbb{P}}$ dependence of the cross section, let us mention that the momentum dependence of the $q \bar{q} g$ final state is quite analogous to that of the $q \bar{q}$ system. We do not show 
the analogue of Fig.2: it is again essential that the two gluons couple to the diffractive system in all possible ways. As before, one has to start in the region where the transverse momenta of all three partons are large. When trying to integrate over the transverse momentum of the gluon, one finds dominance by the low-momentum region: in this region, all nonplanar couplings of the two gluon lines of the Pomeron to the diffractive state become less important, and we are left with the leading-twist 'Pomeron structure function' picture, where the Pomeron interacts only with the gluon and not the quarks.

Next we adress the $x_{\mathbb{P}}$ dependence of the cross section. So far we have drawn a rather simple picture of the diffractive final state: both for the $q \bar{q}$ and the $q \bar{q} g$ final state, we have argued that in the preferred configuration at least one of the final-state partons has a rather soft transverse momentum, and it is this parton which couples to the Pomeron. If the virtuality of this parton is characterized by a typical hadronic scale $\sim \Lambda_{Q C D}$, this seems to imply that the energy dependence of the diffractive cross section should be the same as in hadron-hadron scattering, i.e., the diffractive structure function $F_{2}^{D}$ grows as $\left(1 / x_{\mathbb{P}}\right)^{n_{\mathbb{P}}}$ with $n_{\mathbb{P}}=2 \alpha_{\mathbb{P}}(0)-1 \approx 1.12$. However, because of the observed rise of the gluon structure function at small $x$, the situation is more complicated. Let us return to the above discussion of the $q \bar{q}$ final state. The perturbative region is that of large transverse momenta of the final-state partons. For this part of the phase space, we expect the Pomeron to be described by the perturbative two-gluon model, i.e., the $x$ dependence of the cross section will be given by the square of the gluon structure function of the proton [13]:

$$
\frac{d \sigma}{d M^{2} d t d k_{t}^{2}} \sim\left[x_{\mathbb{P}} g\left(x_{\mathbb{P}}, k_{t}^{2} /(1-\beta)\right)\right]^{2}
$$

This should lead to a rise $F_{2}^{D} \sim\left(1 / x_{\mathbb{P}}\right)^{n_{\text {hard }}}$ where $n_{\text {hard }}=2 \alpha_{\text {hard }}-1$ grows with the transverse momentum $k_{t}^{2}$ of the partons, and typically lies above the value 1.4. For the kinematic region where the quark transverse momenta are small and our perturbative two-gluon Pomeron has to be replaced by some model for the nonperturbative Pomeron, we expect a smaller exponent $n$ : the conventional soft Pomeron would suggest that $n=n_{\mathbb{P}}=2 \alpha_{\mathbb{P}}(0)-1 \approx 1.12$. Since in the diffractive cross section we integrate over both the perturbative and nonperturbative parts of the phase space, there will be competition between the two regions. At first sight, the large-momentum region seemed to be rather subdominant. However, the large gluon structure function provides an enhancement of this region, and in this way weakens the dominance of the soft nonperturbative region. As a result, the effective value of the exponent $n, n_{e f f}$, is expected to lie somewhere between the soft and the hard values, and the effective scale at which the $k_{t}$ integral peaks should be somewhat higher than the soft Pomeron scale. Theoretical studies [19] indicate that $n_{\text {eff }}$ only weakly depends upon $Q^{2}$, but they do not allow us to predict the numerical value of $n_{\text {eff }}$ or the momentum scale.

\subsection{The Parametrization}

After this brief theoretical review, we are ready to describe and motivate our parametrization. It will be given in terms of the diffractive structure function $F_{2}^{D}$, and can be written as the sum of several distinct contributions. In our fit we include the following four pieces:

$$
F_{2}^{D}=F_{q \bar{q}}^{T}+F_{q \bar{q} g}^{T}+\Delta F_{q \bar{q}}^{L}+\Delta F_{q \bar{q}}^{T} .
$$

Here the first and the second term, as indicated by the subscripts and by the superscripts, denote the production of a quark-antiquark pair and the production of a quark-antiquark-gluon system with transversely-polarized photons. The third term takes into account the production of a quark-antiquark pair from a longitudinally-polarized photon, and the prefix $\Delta$ indicates that this 
contribution belongs to higher twist (twist four). We have also included a transverse higher-twist contribution to $q \bar{q}$ production, denoted by $\Delta F_{q \bar{q}}^{T}$

Let us discuss these terms in more detail. To begin with the $Q^{2}$ dependence of (11), we recapitulate that in the leading-twist transverse contribution to $q \bar{q}$ production there is no $\log \left(Q^{2} / Q_{0}^{2}\right)$ enhancement from the phase-space integral, whereas $q \bar{q} g$ production is of higher order in $\alpha_{s}$ and has an $\alpha_{s} \ln \left(Q^{2} / Q_{0}^{2}\right)$ dependence. The third term, the longitudinal cross section of the $q \bar{q}$ final state, belongs to higher twist, and the phase-space integral provides a $\log \left(Q^{2} / Q_{0}^{2}\right)$ enhancement. The reason why this contribution is essential will be discussed below. The longitudinal contribution of the $q \bar{q} g$ final state is again of leading twist, but the logarithm is absent. It is therefore subleading in comparison with the transverse contribution, and will be disregarded in our parametrization.

To get an estimate of the $\beta$ spectrum, we consider the limits $\beta \rightarrow 1$ and $\beta \rightarrow 0$. Contact with the other variables is made through the kinematic relation (6). For the $\beta$ dependence of the longitudinal cross section we can use (10) and (5): for the transverse case the situation is slightly more complicated, and we have to use both (9) and (11), in combination with (5). A more intuitive argument can be derived from the wave functions, and goes as follows. The limit $M \rightarrow 0$, which is the same as $\beta \rightarrow 1$, is related to the small- $k_{t}$ behavior of the cross section. Let us return to the wave function (4). For both of the limits $l_{t} \rightarrow 0$ and $l_{t} \rightarrow \infty$, one finds that when $k_{t}$ approaches zero $D \Psi$ vanishes with the same power in $k_{t}$ as the orginal wave function. (In eq.(3), where the denominator is quadratic in $k_{t}$, the integration over the azimuthal angle of $l_{t}$ leads to the final cancellation of nonvanishing contributions.) This means that characteristic features of the light-cone wave functions $\left(\Psi^{0}, \Psi^{ \pm}, \Psi^{\mu \nu}\right)$ remain unchanged after scattering. We expect that these results also hold for multi-gluon exchange. It is important to note that a single gluon (or photon) exchange, as opposed to the color-singlet two-gluon exchange, leads to a rather different spectrum at $\beta \sim 1$. This is because, instead of the second-order derivative in (4), in this case only the first derivative of the wave function is needed, which, unlike the second derivative, does not vanish when $k_{t}$ approaches zero.

Applying these arguments to our wave functions we find, first for the the transverse quarkantiquark production cross section, that the cross section behaves like $(1-\beta)\left(\Psi^{ \pm} \sim Q \overrightarrow{k_{t}}\right.$ from (1) and $M \sim k_{t}$ from (6)), i.e., it vanishes when $M$ becomes zero. For the second contribution with one gluon in the final state and with the tensor structure $\Psi^{\mu \nu} \sim k_{t}^{\mu} k_{t}^{\nu}-2\left|k_{t}\right|^{2} g_{t}^{\mu \nu}$, the cross section vanishes like $(1-\beta)^{2}$. The subsequent integration over the quark-antiquark final state introduces a further suppression, leading to a $(1-\beta)^{3}$ behavior. Finally, for the wave function (2) of longitudinally-polarized photons, we find that $\Psi^{0} \sim Q$, i.e., the cross section goes to a constant different from zero: this means that near $\beta=1$ the longitudinal cross section dominates and cannot be neglected. The other limit $\beta \rightarrow 0$ or $M \rightarrow \infty$ is dictated by the high-energy behavior of the amplitudes, which is different for quark and gluon exchange. Spin- $1 / 2$ exchange is suppressed relative to spin-1 exchange, which leads to a dominance of gluon production at small $\beta$ over the leading-order quark-antiquark production. We conclude that our three contributions, transverse $q \bar{q}$ and $q \bar{q} g$ production and longitudinal $q \bar{q}$ production, are important in rather distinct regions in $\beta$, namely medium, small, and large $\beta$, respectively. The transverse higher-twist contribution is expected to give a small negative correction which is due to phase space limitations at finite $Q^{2}$ [20].

Finally, we comment again on the energy dependence. In contrast to the $\beta$ spectrum, which can be traced back to rather general properties of the wave functions, perturbative QCD does not allow us to control the $x_{\mathbb{P}}$ dependence of the cross section. In particular, $n_{\text {eff }}$ for the leadingtwist transverse cross sections cannot yet be predicted, and we therefore let the data decide on the preferred values of this exponent. As discussed at the end of the previous subsection, we expect a weak $Q^{2}$ dependence. For the higher-twist longitudinal part, on the other hand, theoretical 
arguments have been given which indicate that the $x_{\mathbb{P}}$ dependence is given by the square of the gluon structure function at the momentum scale $Q^{2} / 4 \beta$, i.e., it should grow with $Q^{2}$. In our fit we assume a universal $x_{\mathbb{P}}$ dependence of all higher-twist terms. The exponent is allowed to vary with $Q^{2}$.

After these remarks, we finally write down our Ansatz for the diffractive structure function. For the four terms in (11) we put:

$$
\begin{aligned}
F_{q \bar{q}}^{T} & =A\left(\frac{x_{0}}{x_{\mathbb{P}}}\right)^{n_{2}} \beta(1-\beta) \\
F_{q \bar{q} g}^{T} & =B\left(\frac{x_{0}}{x_{\mathbb{P}}}\right)^{n_{2}} \alpha_{s} \ln \left(\frac{Q^{2}}{Q_{0}^{2}}+1\right)(1-\beta)^{\gamma} \\
\Delta F_{q \bar{q}}^{L} & =C\left(\frac{x_{0}}{x_{\mathbb{P}}}\right)^{n_{4}} \frac{Q_{0}^{2}}{Q^{2}}\left[\ln \left(\frac{Q^{2}}{4 Q_{0}^{2} \beta}+1.75\right)\right]^{2} \beta^{3}(1-2 \beta)^{2} \\
\Delta F_{q \bar{q}}^{T} & =D\left(\frac{x_{0}}{x_{\mathbb{P}}}\right)^{n_{4}} \frac{Q_{0}^{2}}{Q^{2}} \ln \left(\frac{Q^{2}}{4 Q_{0}^{2} \beta}+1.75\right) \beta^{3}(1-\beta) .
\end{aligned}
$$

The exponents are chosen to have the form

$$
\begin{aligned}
& n_{2}=n_{20}+n_{21} \ln \left[\ln \left(\frac{Q^{2}}{Q_{0}^{2}}\right)+1\right] \\
& n_{4}=n_{40}+n_{41} \ln \left[\ln \left(\frac{Q^{2}}{Q_{0}^{2}}\right)+1\right],
\end{aligned}
$$

and $\alpha_{s}$ is set to 0.25 .

As we have discussed before, the shapes of the $\beta$ spectra are restricted by properties of the light-cone wave functions, and one finds for the parameter $\gamma$ in $F_{q \bar{q} g}^{T}$ the value 3. But, since the H1 analysis in [2] reports a rather hard gluon distribution inside the Pomeron, we allow the parameter $\gamma$ to deviate from the model prediction. For the leading-twist (transverse) cross sections the exponent $n_{2}$ will be left to the fit. We have introduced a simple $Q^{2}$-dependent function, although a weak dependence is expected theoretically. The $x_{\mathbb{P}}$ dependence of the-higher twist contributions $\left(n_{4}\right)$, on the other hand, is expected to be given by the square of the gluon structure function, and in principle we could use the measured gluon structure function. We have inserted an extra $\log \left(Q^{2} /\left(4 \beta Q_{0}^{2}\right)\right)$ in order to simulate the effect of having a structure function. For $n_{4}$ we assume the same functional form as for $n_{2}$, but expect to find a stronger rise with $Q^{2}$ than for the leading-twist contributions. The parameter $x_{0}$ was introduced to minimize any effect from $n_{2}$ and $n_{4}$ on the overall $Q^{2}$ shape. The scale parameter $Q_{0}^{2}$ is taken to be $1 G e V^{2}$.

Finally, we mention that, in principle, one should also allow for higher-twist corrections to $q \bar{q} g$ production. However, even if we assume a universal $x_{\mathbb{P}}$ dependence for all higher-twist pieces, this would increase the number of free parameters. We have found that, with the presently available amount and accuracy of data, it is not yet possible to determine these parameters with sufficient accuracy. Therefore, these higher-twist pieces will not be included in our fit.

\section{$3 \quad$ Fits to the Data}

ZEUS Data: We begin with a fit to the ZEUS data [1], whose results are shown in Table 1 and Fig.4. Some of the parameters have been restricted to a physically meaningful range by imposing 

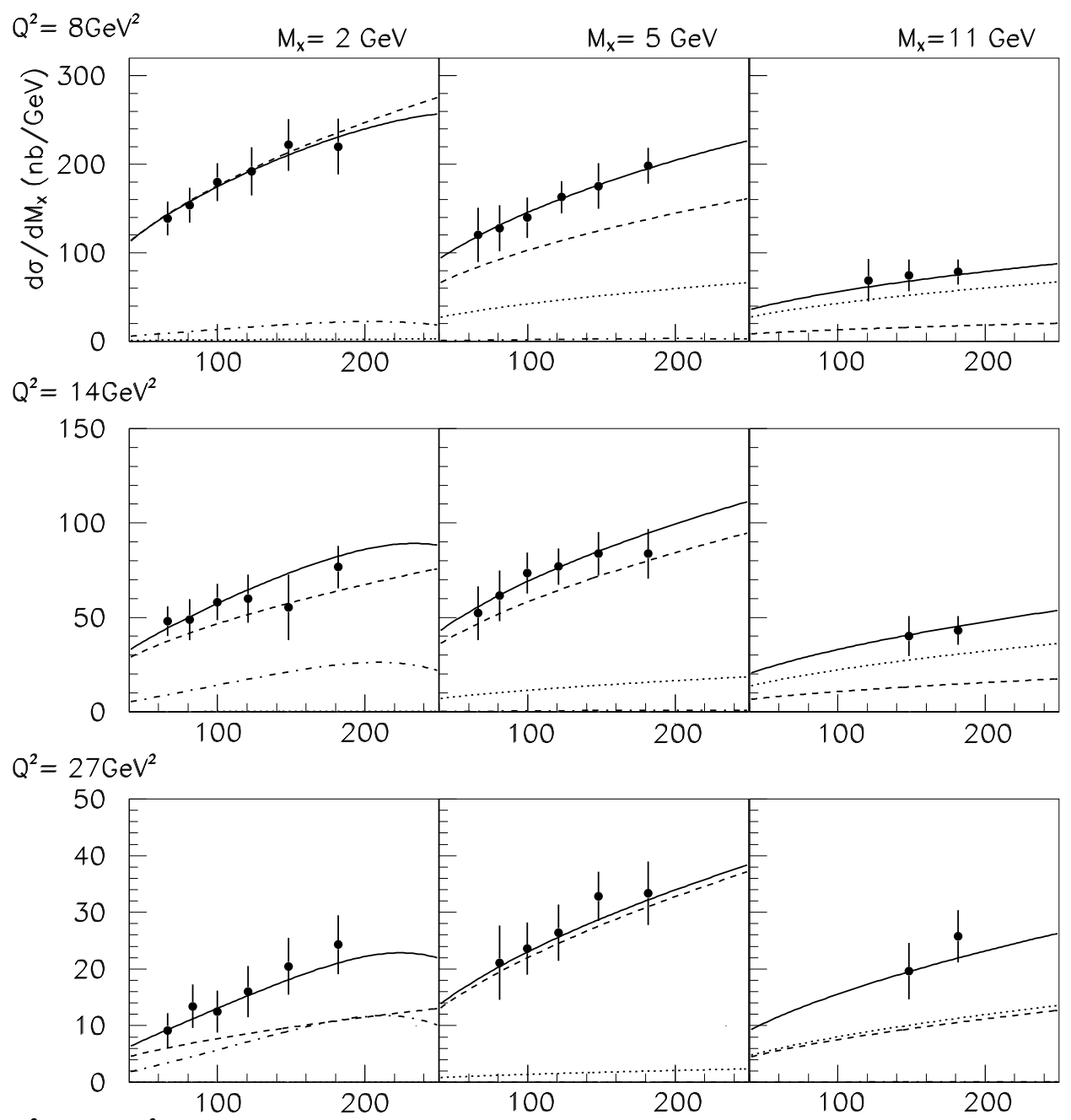

$\mathrm{Q}^{2}=60 \mathrm{GeV}^{2}$

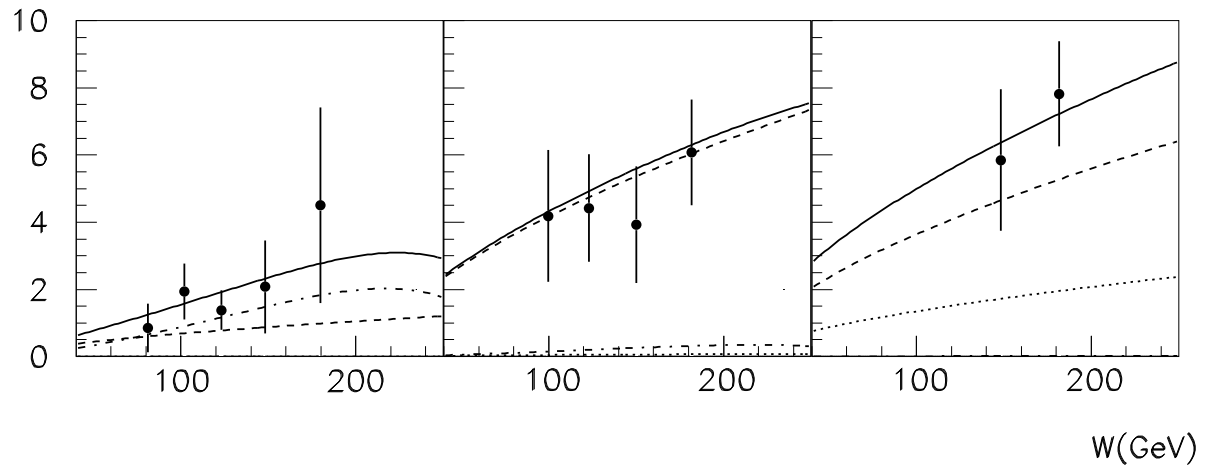

Figure 4: Fit compared to 1994 ZEUS data. Upper solid line: total result, dashed line: $F_{q \bar{q}}^{T}$, dotted line: $F_{q \bar{q} g}^{T}$ and dashed-dotted line: $F_{q \bar{q}}^{L}$. 


\begin{tabular}{|c|c|c|c|c|}
\hline & mean value & error & lower limit & upper limit \\
\hline $\mathrm{A}$ & 293 & 11 & no & no \\
\hline $\mathrm{B}$ & 166 & 25 & no & no \\
\hline $\mathrm{C}$ & 76 & 15 & no & no \\
\hline $\mathrm{D}$ & -184 & 2 & no & no \\
\hline$x_{0}$ & 0.001 & 0.00011 & 0.0001 & 1 \\
\hline$\gamma$ & 4.3 & 0.9 & no & no \\
\hline$n_{20}$ & 1.11 & 0.14 & 1 & 2 \\
\hline$n_{21}$ & 0.12 & 0.12 & 0 & 1 \\
\hline$n_{40}$ & 1 & 0.79 & 1 & 2 \\
\hline$n_{41}$ & 0.43 & 0.12 & 0 & 1 \\
\hline \hline$\chi^{2} /$ d.o.f. & \multicolumn{5}{|c|}{$12 / 43$} \\
\hline
\end{tabular}

Table 1

upper and lower limits. We note that the form of the $q \bar{q} g$ wave function in (3) leads to $\gamma=3$ : our fit, cf. the value of $\gamma$ in Table 1 and the dotted curves in Fig.4, indicates that the data prefer a larger value. The behavior of $F_{q \bar{q} g}$ near $\beta=1$ is therefore far from a 'hard gluon distribution' in the Pomeron. Our fit shows that it is possible to describe the ZEUS data without introducing a hard gluon inside the Pomeron.

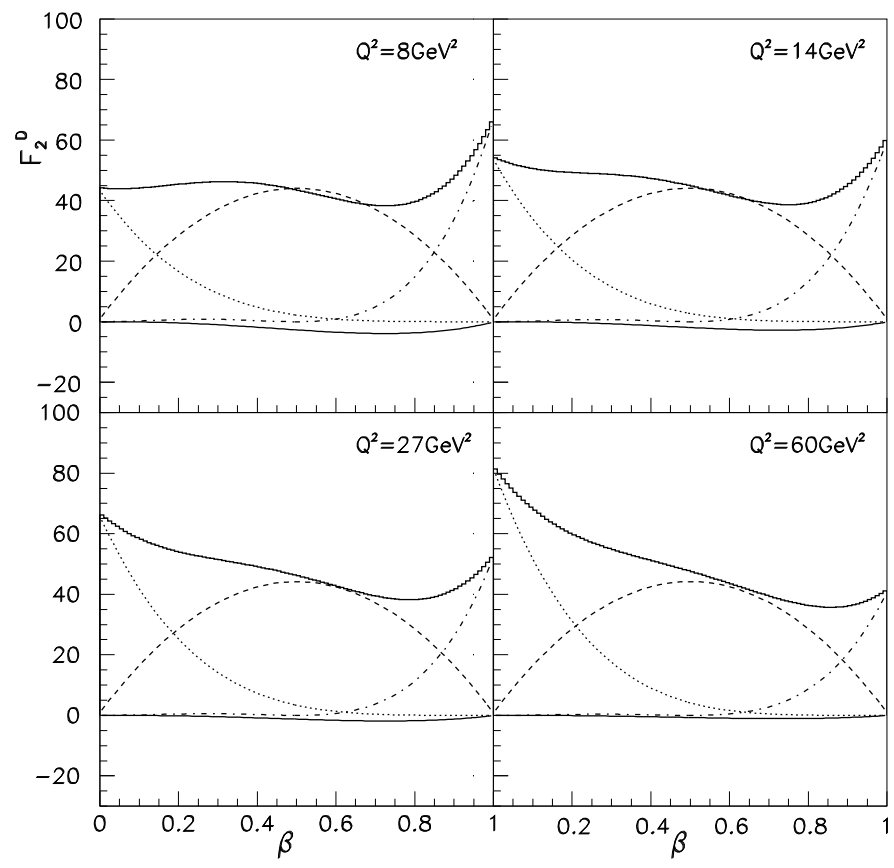

Figure 5: The $\beta$ spectrum at fixed $x_{\mathbb{P}}=0.001$. Upper solid line: total result, dashed line: $F_{q \bar{q}}^{T}$, dotted line: $F_{q \bar{q} g}^{T}$, dashed-dotted line: $F_{q \bar{q}}^{L}$ and lower solid line: $\Delta F_{q \bar{q}}^{T}$.

Fig.5 shows the $\beta$ spectrum. One recognizes the subdivision into three distinct regions: the small- $\beta$ region with $q \bar{q} g$ production, the medium- $\beta$ region with transversely-produced $q \bar{q}$ pairs, and 
the large- $\beta$ region where the longitudinal part dominates. The sum of all three contributions leads to a rather flat $\beta$ spectrum.

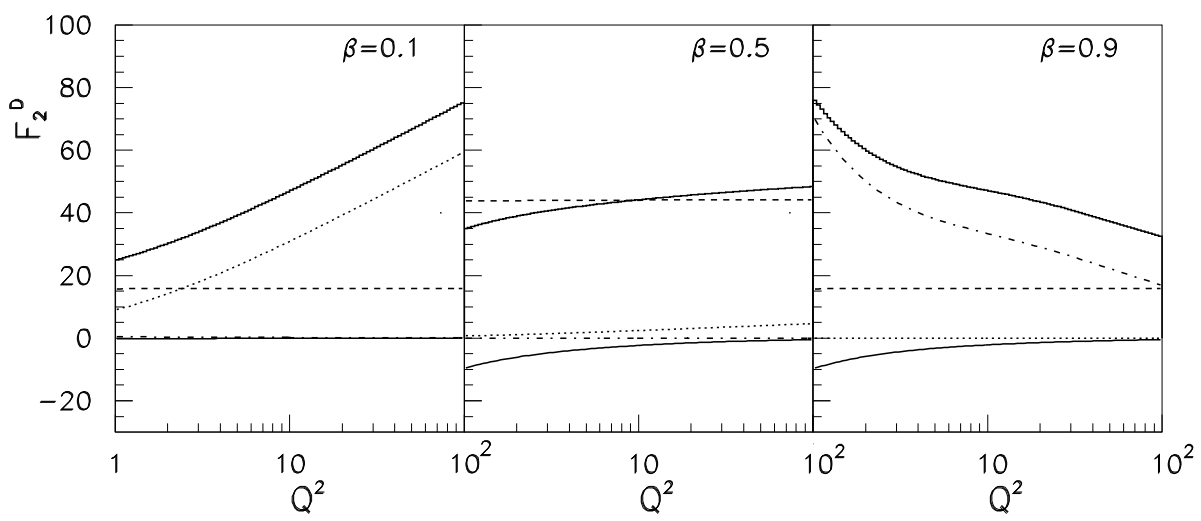

Figure 6: The $Q^{2}$ distribution at fixed $x_{\mathbb{P}}=0.001$. The notations of the lines are the same as in the previous figures.

The $Q^{2}$ distribution in Fig.6 has the expected shape: it is logarithmically increasing at $\beta=0.1$ (which we associate with $q \bar{q} g$ production), constant for the leading-twist transverse part at $\beta=$ 0.5 , and decreasing for the longitudinal part at $\beta=0.9$. The negative slope of the longitudinal contribution, which is due to its higher-twist nature, is partly compensated by the counteracting logarithm in $Q^{2}$. The higher-twist correction for the transverse part, which is also decreasing when the absolute value is considered, leads to a small positive slope at $\beta=0.5$ in the combined result.

The transverse higher-twist correction to $q \bar{q}$ production wants to be negative, as expected from theoretical arguments [20]. Its absolute magnitude is rather small, as can be seen in Figs.5 and 6 , and it represents a correction to the three leading pieces. As mentioned before, we have also attempted to include higher-twist corrections to the $q \bar{q}$ production: with the available statistics it is not possible to assert whether such a contribution is present or not.

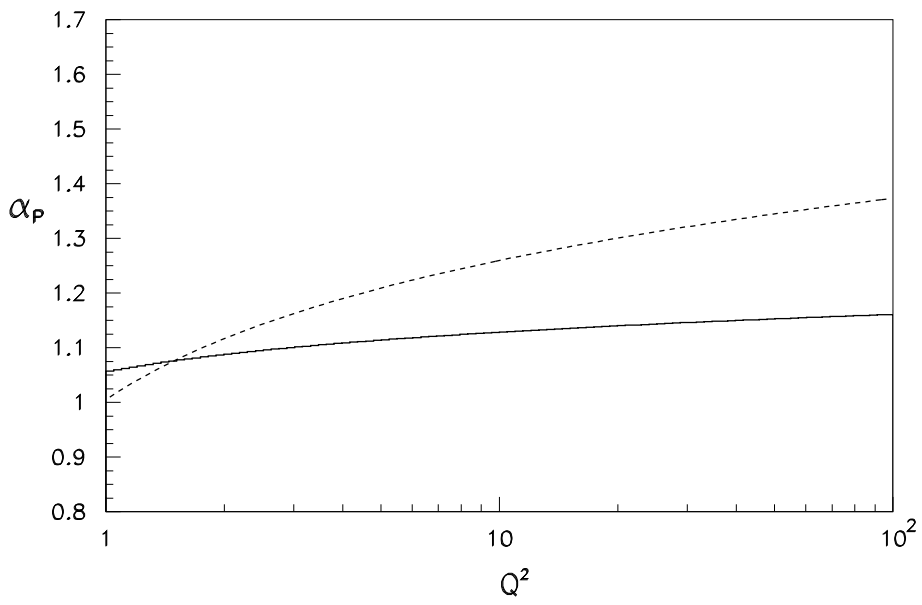

Figure 7: Pomeron intercept for leading twist (solid line) and higher twist (dashed line). 
Although some of the parameters $n_{2} 0, \ldots, n_{41}$ have substantial errors, their values, as determined from the fit, lead to an appealing theoretical scenario. The value $n_{20}=1.11$ corresponds to an effective Pomeron intercept $\alpha_{\mathbb{P}}(0)=1.055$, which is consistent with the 'soft' Pomeron of Donnachie and Landshoff. It exhibits, however, a slight rise with $Q^{2}$, which suggests that in the leading-twist transverse cross section the Pomeron is already a mixture of the soft Pomeron and the gluon structure function. According to our discussion above, this means that the effective momentum scale of the aligned-jet configuration in $q \bar{q}$ production is slightly higher than the typical hadronic scale of the soft Pomeron. The intercept for the higher-twist contribution (the dashed line in Fig. 7), on the other hand, shows a strong rise with increasing $Q^{2}$. It tends towards a value of 1.2 at large $Q^{2}$, which is compatible with the gluon structure function, i.e., the Pomeron is hard in this case.

It is also interesting to compare the ZEUS fit with data from H1. Fig.8 shows all H1 data points with the ZEUS fit result overlaid. The agreement is quite good when all points above $x_{\mathbb{P}}=0.01$ are ignored. The strongest deviation is observed in the $\beta=0.2$ bin at low $Q^{2}$. Here the ZEUS fit lies considerably above the $\mathrm{H} 1$ data. The overall impression, however, is that in the kinematic range where the Pomeron dominates over secondary trajectories, both data sets are consistent. We find remarkable the good agreement between the H1 data and the ZEUS fit at $\beta=0.9$ and low $Q^{2}$, since this region is not covered by ZEUS data. The fit seems to provide here a good extrapolation. In summary, we have shown that it is possible to describe the ZEUS data using the first three terms in (12), the fourth term providing a small correction. In particular, there is no need for a hard gluon inside the Pomeron. Furthermore, our fit is quite consistent with our theoretical expectations: the parameter $\gamma$ as well as the exponents $n_{2}$ and $n_{4}$ have chosen values which seem to confirm the ideas outlined in the previous section.

H1 Data: Next we turn to the H1 data and describe the results of our fit to them. Most remarkable is the fact that we find two different solutions. The parameters of the first solution are given in Table 2, and the corresponding comparison of fit and data can be found in Fig.9.

\begin{tabular}{|c|c|c|c|c|}
\hline & mean value & error & lower limit & upper limit \\
\hline $\mathrm{A}$ & 16.8 & 8.4 & no & no \\
\hline $\mathrm{B}$ & 13.9 & 4.2 & no & no \\
\hline $\mathrm{C}$ & 10.5 & 2.6 & no & no \\
\hline $\mathrm{D}$ & 0 & 6.5 & no & no \\
\hline$x_{0}$ & 0.0033 & 0.00063 & 0.0001 & 1 \\
\hline$\gamma$ & 0.28 & 0.08 & no & no \\
\hline$n_{20}$ & 1 & 0.14 & 1 & 2 \\
\hline$n_{21}$ & 0.19 & 0.024 & 0 & 1 \\
\hline$n_{40}$ & 1.6 & 0.17 & 1 & 2 \\
\hline$n_{41}$ & 0 & 0.82 & 0 & 1 \\
\hline \hline$\chi^{2} /$ d.o.f. & \multicolumn{5}{|c|}{$139 / 130$} \\
\hline
\end{tabular}

Table 2

In order to avoid secondary trajectories, we have imposed an upper cut on $x_{\mathbb{P}}$ of 0.01 . A striking feature of this solution is the fact that the exponent $\gamma$ is well below 1 , which can be interpreted as implying an initial gluon distribution that is singular for $\beta=1$. The explanation for this result can be inferred from Fig.10, where we have compiled all relevant diagrams, i.e., the $\beta$ spectrum, 


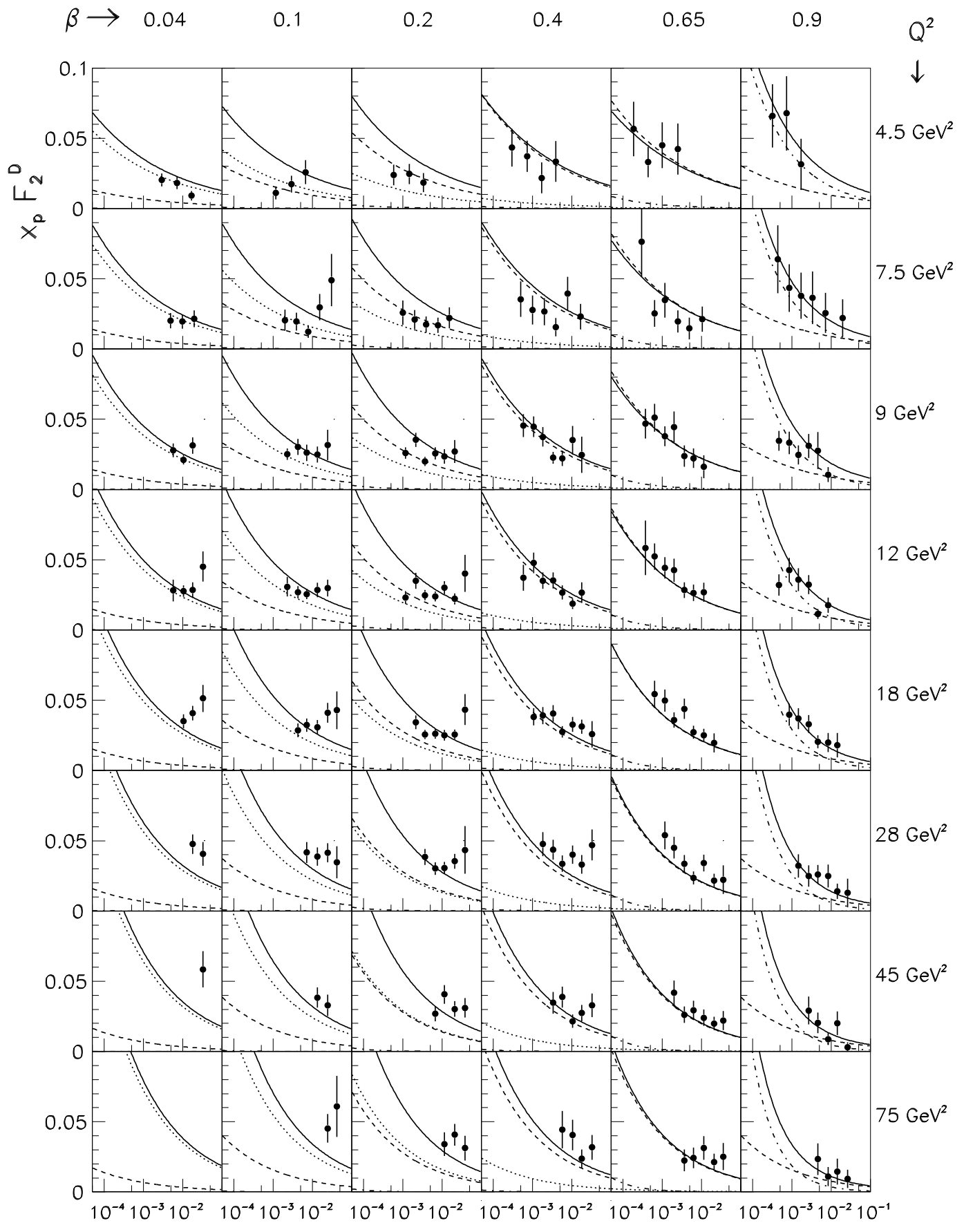

Figure 8: The ZEUS fit compared to H1 data. Upper solid line: total result, dashed line: $F_{q \bar{q}}^{T}$, dotted line: $F_{q \bar{q} g}^{T}$ and dashed-dotted line: $F_{q \bar{q}}^{L}$. 


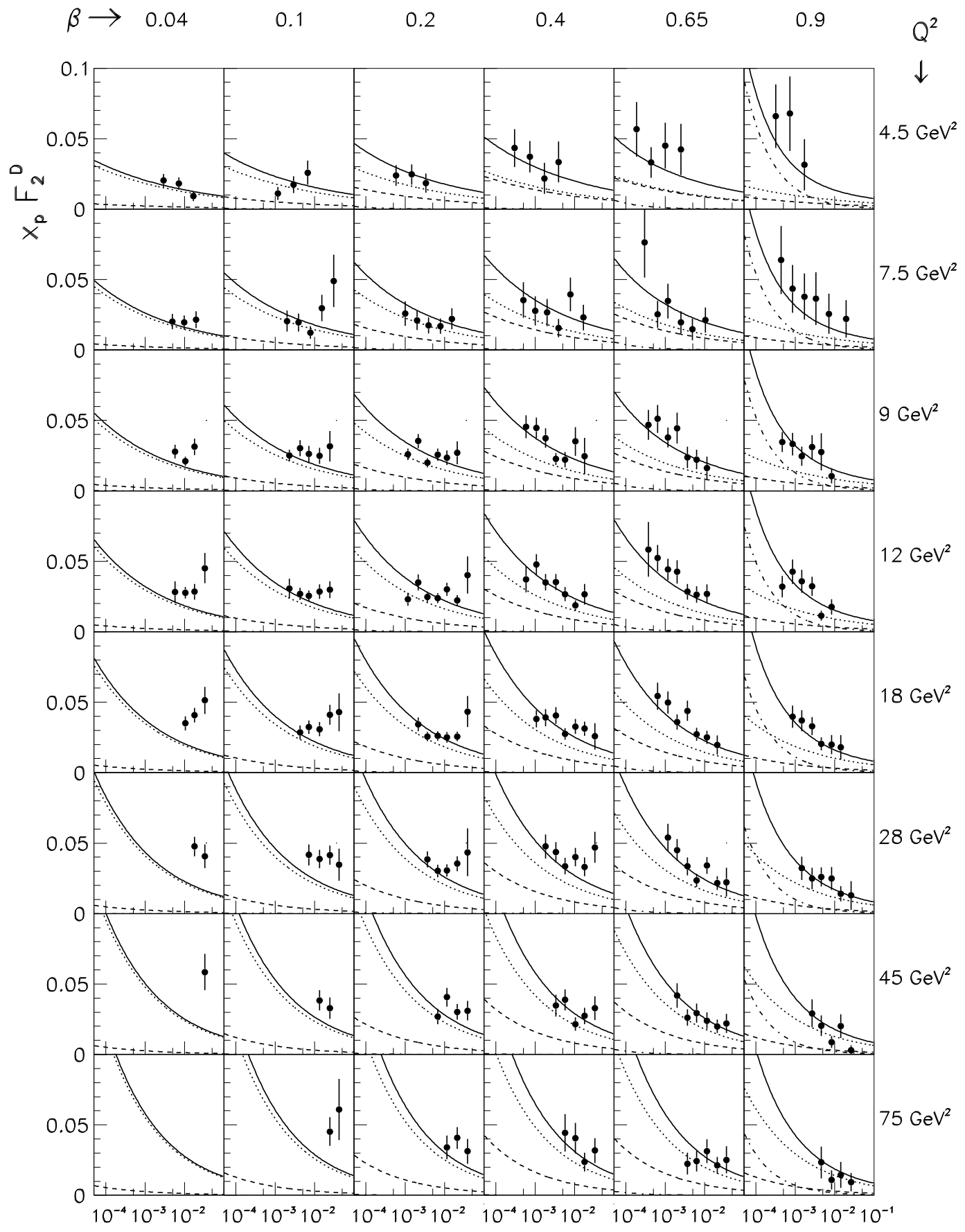

Figure 9: The H1 fit compared to H1 data. Upper solid line: total result, dashed line: $F_{q \bar{q}}^{T}$, dotted line: $F_{q \bar{q} g}^{T}$ and dashed-dotted line: $F_{q \bar{q}}^{L}$. 
the $Q^{2}$ dependence and $\alpha_{\mathbb{P}}$, in one figure.
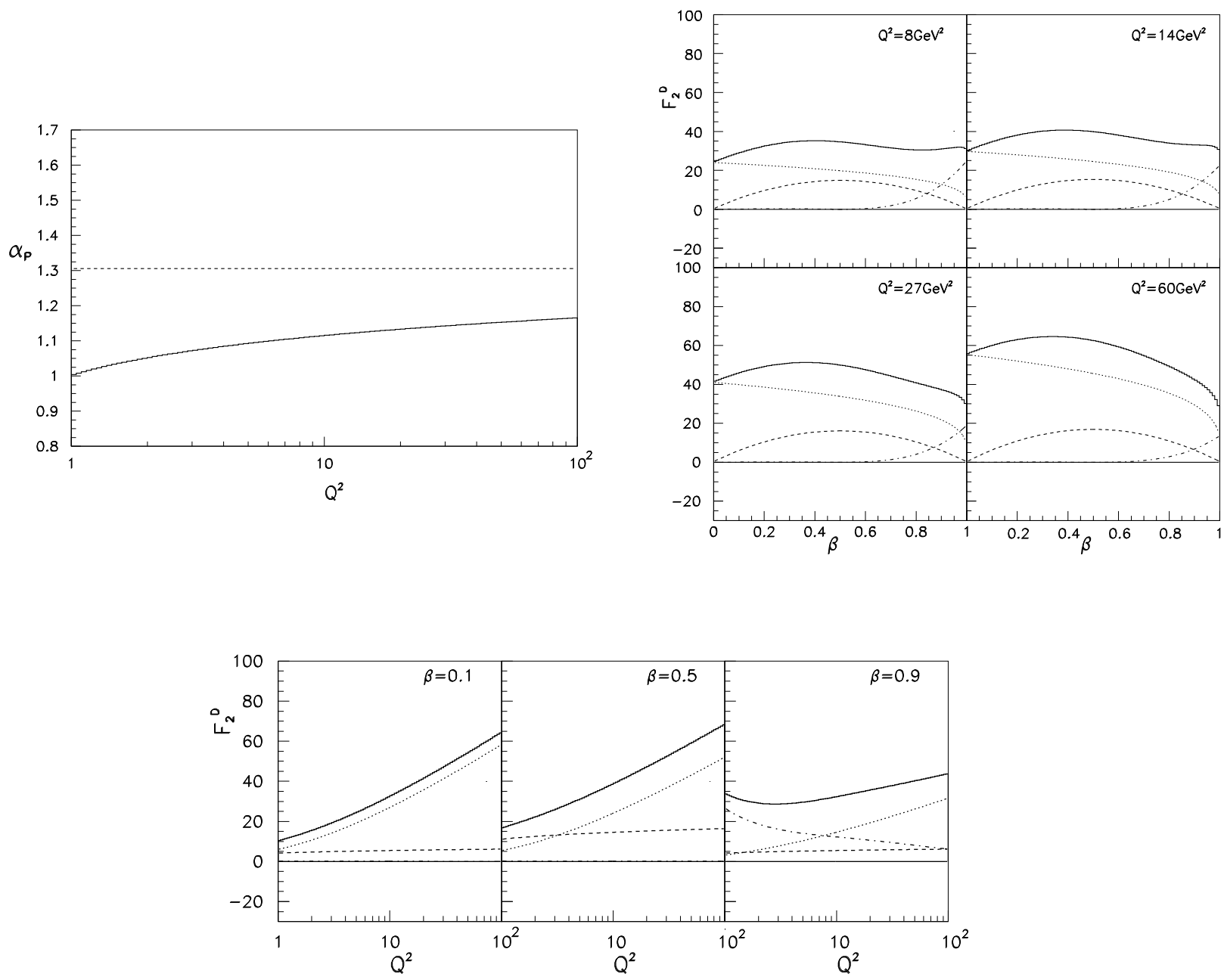

Figure 10: The H1 fit results, in the same notation as in previous figures.

The $\mathrm{H} 1$ data prefer a positive slope in $Q^{2}$, even at a large $\beta$, of 0.5 . With the present parametrization, this can only be achieved by making the gluon contribution $\left(F_{q \bar{q} g}^{T}\right)$ large. Of course, our rather simple approach is not competitive with the more sophisticated analysis that has been performed by H1 [2]. Nevertheless, it mimics the effect of an evolving singular gluon distribution rather well. The $\beta$ spectrum in Fig.10 shows the dominance of the $q \bar{q} g$ contribution over the transverse $q \bar{q}$ contribution, which also spreads into the large- $\beta$ region. The longitudinal contribution has not disappeared completely, but is roughly a factor 2 smaller than in the fit to the ZEUS data. Since the $q \bar{q} g$ contribution has only a rather low Pomeron intercept associated with leading twist, the intercept for the higher-twist contribution is forced to a very high value of 1.3 , in order to accommodate the data. It is completely flat, because we demanded a positive slope in our fit. Without a lower limit on $n_{41}$, the slope would become negative.

Comparing with the ZEUS data, we find again reasonable agreement between the two data sets. The most significant deviation is found for the lower $Q^{2}$ bins. We point out that, when one fits the 

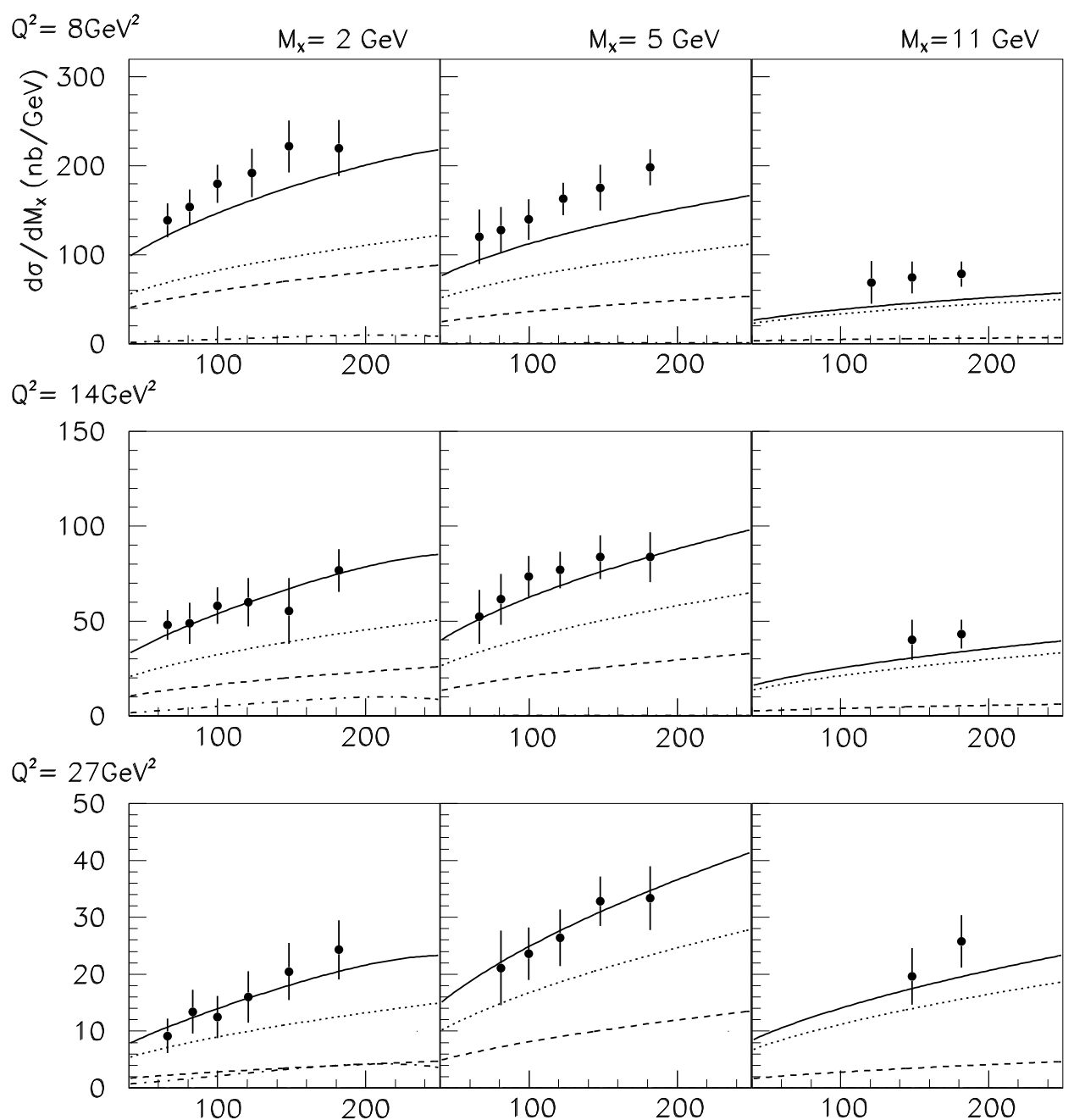

$\mathrm{Q}^{2}=60 \mathrm{GeV}^{2}$

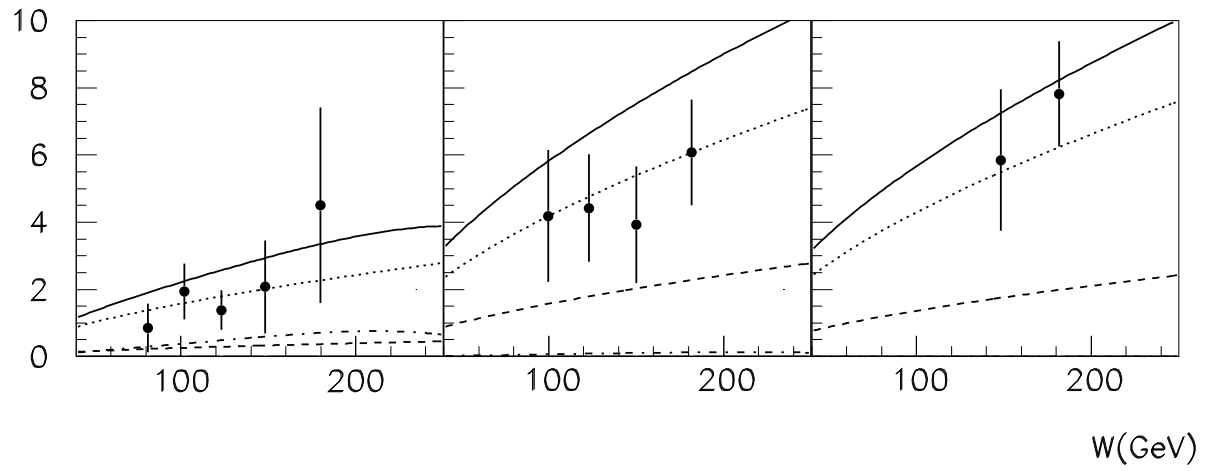

Figure 11: The H1 fit compared to ZEUS data. Upper solid line: total result, dashed line: $F_{q \bar{q}}^{T}$, dotted line: $F_{q \bar{q} g}^{T}$ and dashed-dotted line: $F_{q \bar{q}}^{L}$. 
ZEUS data starting from the H1 data as input, the parameter values always move back towards those found in the earlier fit, i.e., those in Table 1. In particular, the ZEUS data do not favour a value of $\gamma$ smaller than 1 . If one requires a small value of $\gamma$, one obtains an acceptable fit only with an unreasonably small value for $x_{0}$. If, in addition, $x_{0}$ is fixed at, say, $10^{-3}$, the $\chi^{2}$ value of the fit is substantially increased.

As we have already said, the $\mathrm{H} 1$ fit has a second local minimum for a large $\gamma$ of 8.5 . The $\chi^{2}$ is not much worse than in the previous fit (150 compared to 139). The parameters if this solution are given in Table 3, and the comparison with data is shown in Figs.12 and 13 . The $\beta$ and $Q^{2}$ distributions are shown in Fig.14. On the whole, the distributions look similar to the ZEUS fit. In more detail, however, there are a few differences: the $Q^{2}$ shape for $\beta=0.5$ is completely flat, and the $Q^{2}$ dependence of $n_{4}$ is also flat.

\begin{tabular}{|c|c|c|c|c|}
\hline & mean value & error & lower limit & upper limit \\
\hline $\mathrm{A}$ & 1865 & 451 & no & no \\
\hline $\mathrm{B}$ & 1024 & 1.41 & no & no \\
\hline $\mathrm{C}$ & 422 & 1.41 & no & no \\
\hline $\mathrm{D}$ & 0 & 7.11 & 0 & no \\
\hline$x_{0}$ & 0.0002 & 0.000012 & 0.0001 & 1 \\
\hline$\gamma$ & 8.55 & 0.8 & no & no \\
\hline$n_{20}$ & 1.16 & 0.026 & 1 & 2 \\
\hline$n_{21}$ & 0 & 0.047 & 0 & 1 \\
\hline$n_{40}$ & 1.44 & 0.093 & 1 & 2 \\
\hline$n_{41}$ & 0 & 0.11 & 0 & 1 \\
\hline \hline$\chi^{2} /$ d.o.f. & \multicolumn{5}{|c|}{$150 / 130$} \\
\hline
\end{tabular}

Table 3

Summarizing the H1 fit, we find when fitting our model to the H1 data a solution which allows for the singular gluon interpretation, but there is also a second solution that is close to our model, i.e., consistent with the interpretation described in the context of the ZEUS data. Since the $\chi^{2}$ values of both solutions are not that different from each other, one has to search for further consistency checks: more decisive tests might be provided by comparisons with the vectormeson production cross section or with hard diffractive jets. A singular gluon would give a strong transverse component, whereas in our model the longitudinal component is more pronounced. 


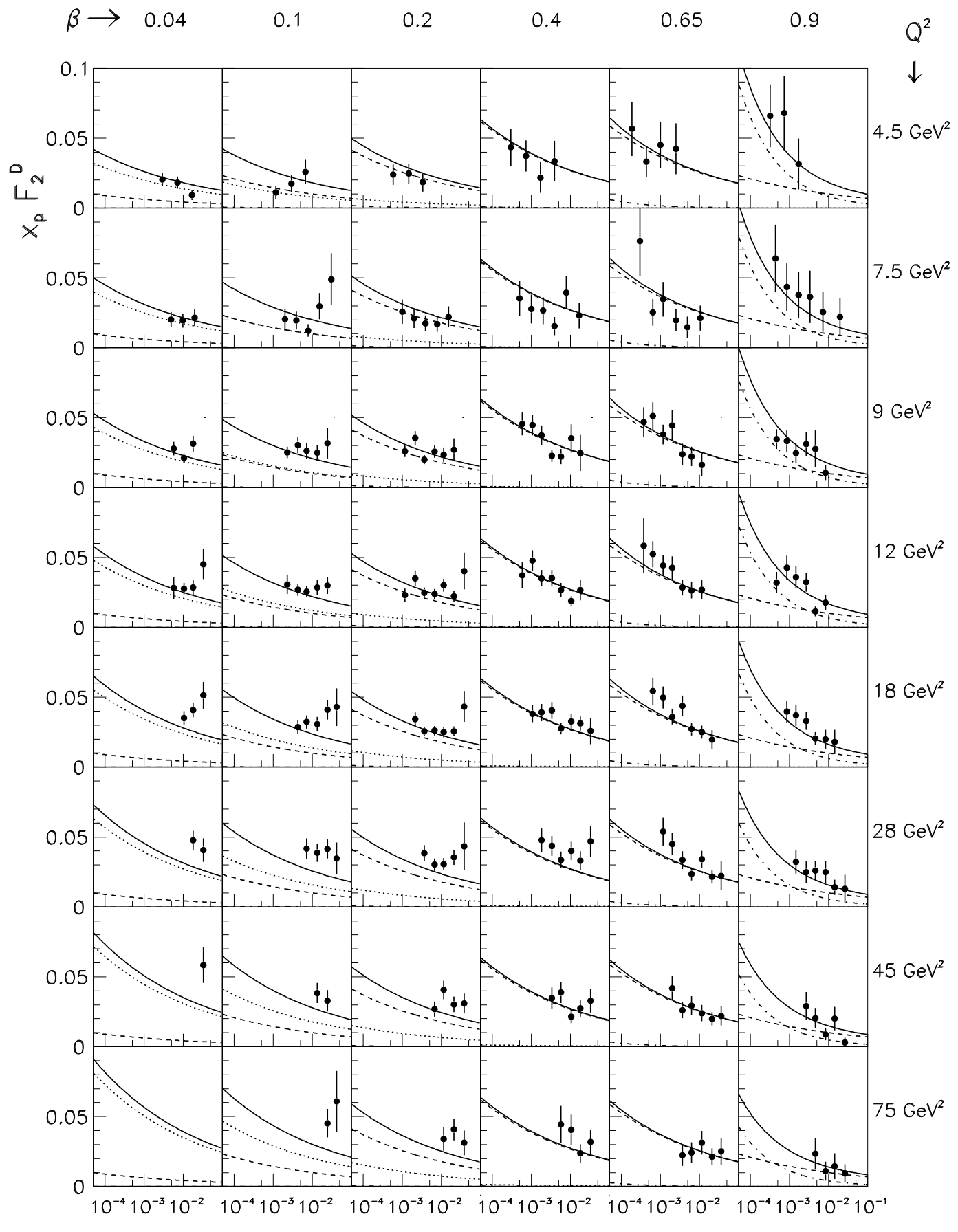

Figure 12: The second H1 fit, with the same notations as before. 

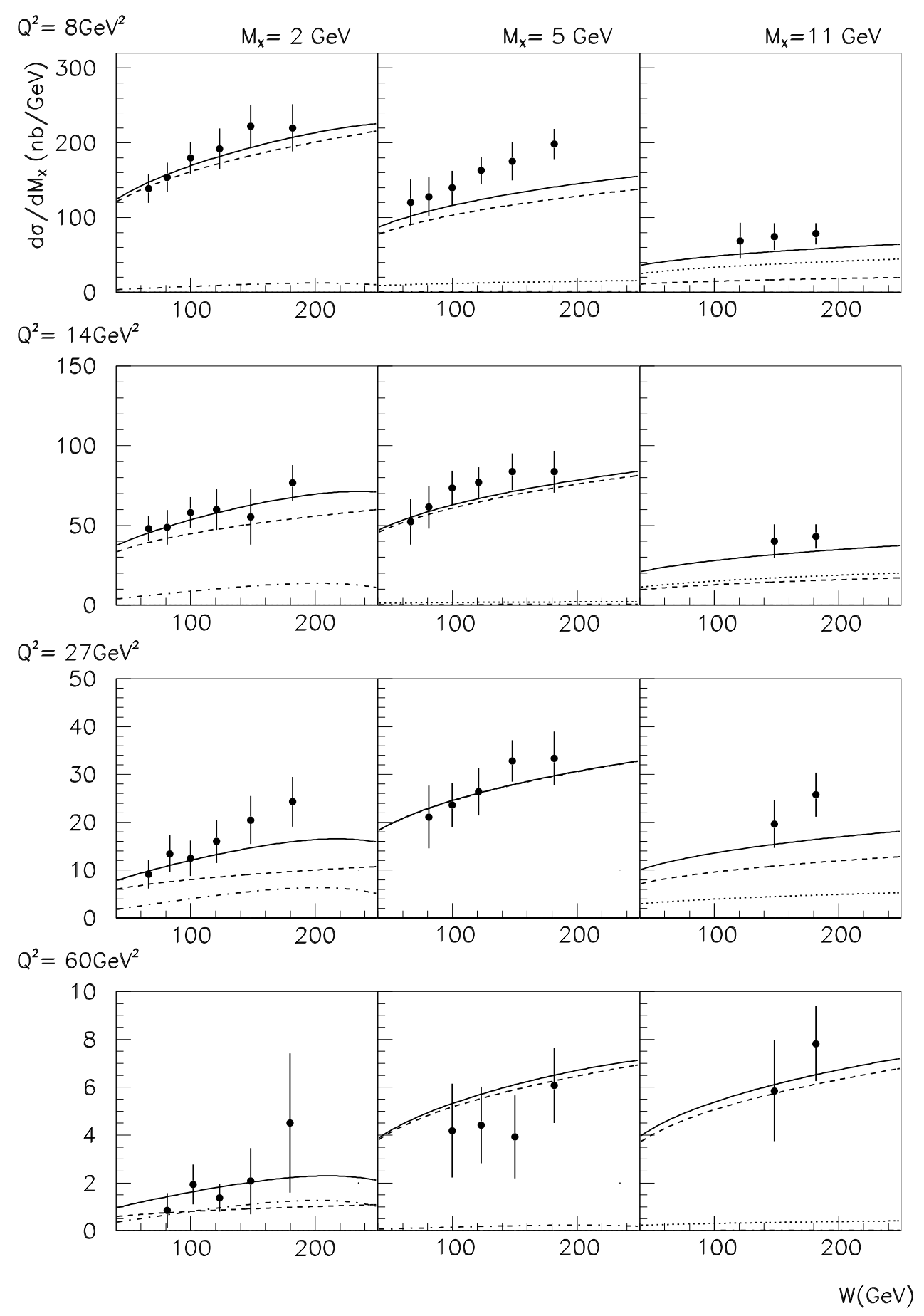

Figure 13: The second H1 fit compared to the ZEUS data. 

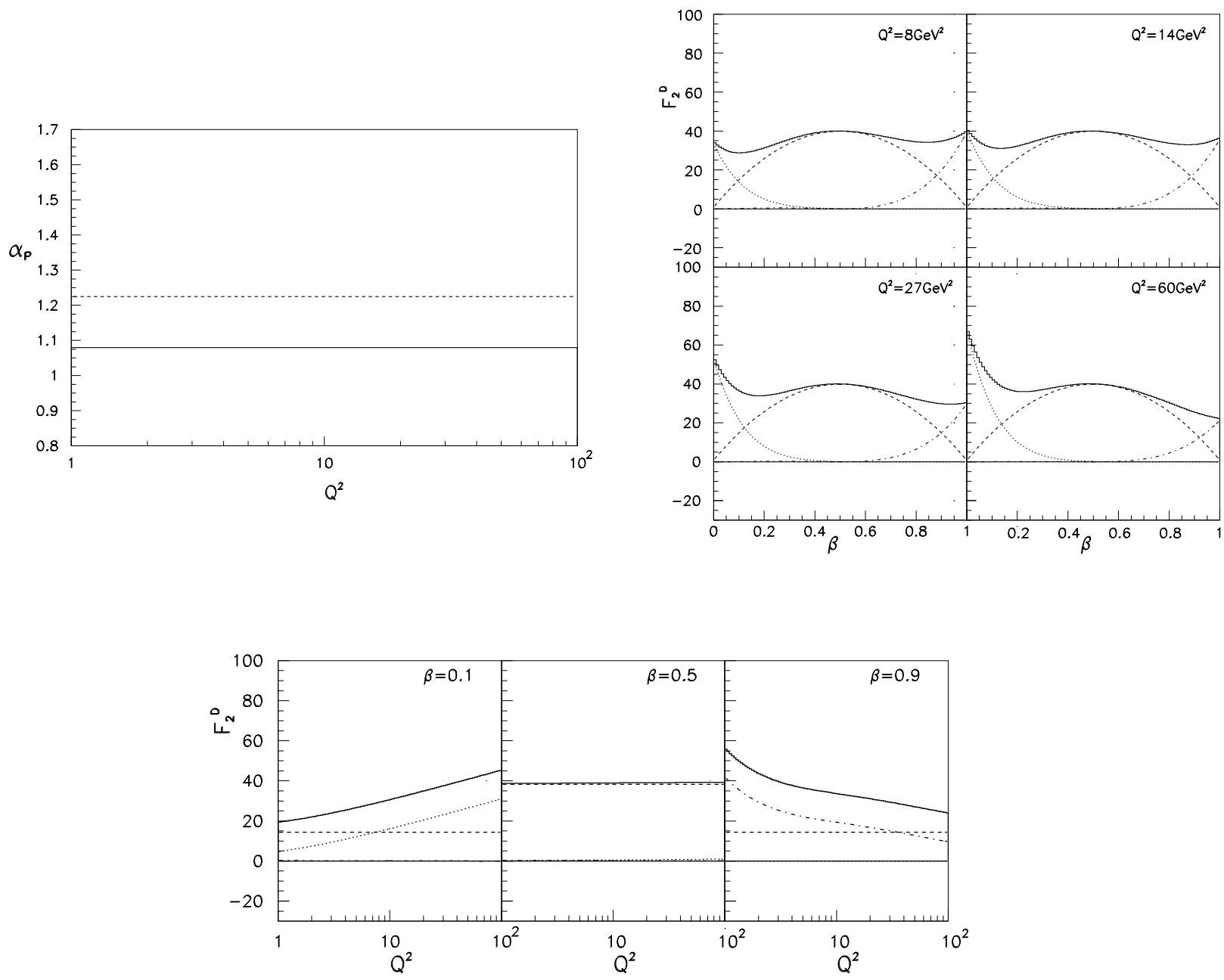

Figure 14: The results of the second H1 fit.

\section{Conclusions}

In this analysis of diffraction in deep-inelastic scattering at HERA, we have proposed a simple parametrization. Working in the wave-function formalism and starting from the 'hard part' of the diffractive cross section, we have suggested a simple extrapolation into the 'soft' nonperturbative region. The main feature of the parameterization is the decomposition of the $\beta$ spectrum into three contributions which reside in separate regions with only little overlap: $q \bar{q} g$ production at low $\beta$, transverse $q \bar{q}$ production at medium $\beta$, and longitudinal $q \bar{q}$ production at large $\beta$. These can be derived from the corresponding wave functions for the $q \bar{q} g$ and $q \bar{q}$ Fock states. The longitudinal contribution is higher twist, whereas the other two contributions are leading twist. The very different $\beta$ dependence of these three contributions allows to determine their relative importance through the fits to the data. We fitted therefore our model to the recent ZEUS and H1 data.

An important result of the fit to the ZEUS data is the dominance of the Pomeron-quark coupling at larger $\beta$ values $(\beta>0.3)$. The Pomeron-gluon coupling becomes substantial at lower $\beta(\beta<0.3)$. The region of large $\beta(\beta>0.9)$ is dominated by the longitudinal contribution. 
The fit to the $\mathrm{H} 1$ data leads to two solutions. In order to be open towards the H1 conjecture of a singular gluon distribution at $\beta=1$ [2], we have allowed, in the second term of our parametrization, the exponent $\gamma$ to be variable. A singular gluon distribution would predict $\gamma<1$, whereas our model suggests $\gamma=3$, or even a little bit higher when $Q^{2}$ evolution is included. Whereas the ZEUS fit found only the option of a large $\gamma$ value, the two H1 solutions have very different $\gamma$ values. In the first fit, $\gamma$ takes a small value, consistent with the H1 conjecture. The Pomeron couples predominantly to gluons and only weakly to quarks. In the second solution, which has almost the same probability ( $\chi^{2}$ value) as the first one, $\gamma$ is much larger and the Pomeron couples mostly to quarks. The coupling to gluons becomes substantial only at low $\beta$, quite similar to the ZEUS fit.

So far one cannot draw a final conclusion about the correct interpretation of both data sets. On the one hand, the conjecture of a singular gluon seems unlikely, in view of the ZEUS data. On the other hand, our parametrization with the two-gluon exchange as a model for the Pomeron can describe both data sets. More insight into the final state is needed, which could, for example, be provided by careful analyses of vector meson production and/or diffractively-produced jets.

\section{References}

[1] ZEUS Collab., H.Kowalski, Talk presented at the LISHEP98 - LAFEX International School on High-Energy Physics, Feb. 16 - 20, 1998, Rio de Janeiro, Brasil.

[2] H1 Collab., DESY 97-158 and Z.Phys. C76: 613 (1997).

[3] F.E. Low, Phys.Rev. D12: 163 (1975); S. Nussinov, Phys.Rev.Lett 34: 1286 (1975).

[4] J. Bartels and M. Wüsthoff, J.Phys. G: Nucl.Part.Phys.22: 929 (1996).

[5] A. Donnachie and P.V. Landshoff, Nucl.Phys. B303: 634 (1988); Nucl.Phys. B244: 322 (1984).

[6] N. Nikolaev and B.G. Zakharov, Z.Phys. C53: 331 (1992).

[7] M. Diehl, Z.Phys. C66: 181 (1996).

[8] E. Gotsman, E. Levin and U. Maor, Nucl.Phys. B493: 354 (1997).

[9] W. Buchmueller and A. Hebecker, Phys.Lett. B355: 573 (1995); Nucl.Phys. B476: 203 (1996); W. Buchmueller, M.F. McDermott and A. Hebecker, Nucl.Phys. B487: 283 (1997), Erratum - ibid. B500: 621 (1997).

[10] M. Wüsthoff, Phys.Rev. D56: 4311 (1997).

[11] A.H. Mueller, Nucl.Phys. B415: 373 (1994), Nucl.Phys. B335: 115 (1990).

[12] S.J. Brodsky, L. Frankfurt, J.F. Gunion, A.H. Mueller and M. Strikman, Phys.Rev. D50: 3134 (1994).

[13] J. Bartels, H. Lotter and M. Wüsthoff, Phys.Lett. B379: 239 (1996), Erratum - ibid. B382: 449 (1996).

[14] J.Bartels and M.Wüsthoff, in preparation.

[15] J. Ellis and G.G. Ross, Phys.Lett. B384: 293 (1996).

[16] K. Golec-Biernat, J. Kwiecinski, Phys.Lett. B353: 329 (1995). 
[17] H.G. Kohrs, DESY-95-248, International Workshop on Deep-Inelastic Scattering and QCD (DIS 95), Paris, France, 24-28 April 1995.

[18] T. Gehrmann and W.J. Stirling, Z.Phys. C70: 89 (1996).

[19] J.Bartels, H.Lotter, and M.Wüsthoff, Z.Phys. C 68: 121 (1995)

[20] J. Bartels and M. Wüsthoff, ANL-HEP-CP-97-51 (1997), International Workshop on DeepInelastic Scattering and QCD (DIS97), Chicago, USA, April 14-18, 1997.

[21] J.D. Bjorken, in: Lecture Notes in Physics 56, Springer Verlag 1975, p.93. 